\title{
Prevalence of O-serogroups, virulence genes, and F18 antigenic variants in Escherichia coli isolated from weaned piglets with diarrhea in Korea during 2008-2016
}

\author{
Kyung-Hyo Do ${ }^{1}$, Jae-Won Byun ${ }^{2}$, Wan-Kyu Lee ${ }^{1, *}$ \\ ${ }^{1}$ Laboratory of Veterinary Bacteriology and Infectious Diseases, College of Veterinary Medicine, Chungbuk National University, Cheongju 28644, Korea \\ ${ }^{2}$ Animal Disease Diagnostic Division, Animal and Plant Quarantine Agency, Gimcheon 39660, Korea
}

\begin{abstract}
To diagnose colibacillosis, detection of O-serogroups and virulence genes has been recommended worldwide. The prevalence of virulence factors can fluctuate over time. The objectives of this study were to determine the prevalence of O-serogroups, virulence genes, and F18 subtypes among pathogenic Escherichia coli isolated from weaned piglets with diarrhea in Korea. Between 2008 and 2016,362 E. coli were isolated from weaned piglets with diarrhea. Hemolysis was determined in blood agar, and O-serogroups were identified using the slide agglutination technique. The genes for the toxins and fimbriae were amplified by polymerase chain reaction (PCR). Real-time PCR was conducted to discriminate between F18 subtypes. Although the most prevalent serogroup was O149 (11.3\%) in the last 9 years, O139 (19.1\%) became the most prevalent in recent years (2015-2016). The most predominant pathotype was enterotoxigenic $E$. coli $(61.3 \%)$. The frequencies of Shiga-like toxin-producing E. coli (STEC) (23.4\%), O139 (19.1\%), Stx2e (35.1\%), and F18ab (48.7\%) increased over the most recent years. Although enterotoxigenic E. coli was the most predominant pathotype, the frequencies of O139, Stx2e, STEC, and F18ab have increased in recent years. These results demonstrate that there have been temporal changes in the predominant O-serogroups and virulence genes over the last decade in Korea. These findings can be practicable for use in epidemiology and control measures for enteric colibacillosis in Korean piggeries.
\end{abstract}

Keywords: Edema disease; Escherichia coli; Post-weaning diarrhea; Virulence profiles; Weaned piglets

\section{Introduction}

Post-weaning diarrhea (PWD) and edema disease (ED) in weaned piglets cause serious economic losses due to diarrhea, growth retardation, and mortality [12,15]. Enterotoxigenic Escherichia coli (ETEC) and Shiga-like toxin-producing E. coli (STEC) are important causes of PWD and ED, respectively. Their pathogenicity involves adherence of the bacteria to the small intestine via specific adhesion factors (fimbriae) and production of several enterotoxins responsible for the disease.

ETEC produces heat-labile (LT) and heat-stable (ST) toxins, and ST toxin is subdivided into STa and STb types [11]. STEC, also called verotoxin-producing $E$. coli, produces the Stx2e variant, which causes systemic vascular damage resulting in ED [18]. In addition, ETEC and STEC can produce one or more fimbriae: F4 (K88), F5 (K99), F6 (987P), F18, and F41 (F7). Fimbriae have a significant role in adhesion to intestinal mucosa and epithelial cells [4].

Although a variety of O-serogroups have been associated with colibacillosis, a limited number of serogroups have been reported in PWD and ED of piglets. The most commonly reported O-serogroups involved in PWD worldwide are O8, O141, and O149. Additionally, the O-serogroups related to ED are $\mathrm{O} 138$ and $\mathrm{O} 139[12,14]$.

F18 fimbriae are usually detected in E. coli isolated from weaned piglets, and they are subtyped into two antigenic variants, F18ab (F107) and F18ac (2134P, 8813) [12,14,15]. F18ab is often associated with strains producing Stx2e, which causes ED, whereas F18ac is associated with ETEC causing PWD [9]. Recently, a new F18 variant (F18new) was identified in E. coli isolated in Germany [2] and Korea [5].

The frequency of these virulence factors is known to vary over time $[8,13,17]$. Identification of the virulence profiles of $E$. coli is important because the information regarding the 
prevalence of pathogenic $E$. coli strains expressing specific fimbriae and enterotoxins is essential for controlling colibacillosis. There are many studies regarding the prevalence of virulence factors in pathogenic $E$. coli $[7,10,21]$, but few studies have examined annual changes in virulence traits of $E$. coli in Korea. The objectives of this study were to determine the prevalence of the O-serogroups, fimbriae, enterotoxins, and F18 subtypes among pathogenic $E$. coli isolated from weaned piglets with diarrhea from 2008 to 2016 .

\section{Materials and Methods}

\section{Escherichia coli isolates}

Between 2008 and 2016, 362 E. coli isolates were obtained from weaned piglets showing symptoms of ED or PWD. The sampled farms consisted of 100 different pig herds (50 to 100 sows per each herd) and were located in three areas: northern (24 farms encompassing the Gangwon, Gyeonggi, and Incheon provinces), middle (26 farms, Chungbuk and Chungnam provinces) and southern (50 farms, Chonbuk, Chonnam, Gyeongbuk, and Gyeongnam provinces) South Korea. Strains were not collected repeatedly from the same farm. The aseptically collected intestinal contents and feces were inoculated on MacConkey agar (BBL; Fisher Scientific, USA) and blood agar (Asan Pharmaceutical, Korea). After overnight incubation at $37^{\circ} \mathrm{C}$, only pure or nearly pure cultured colonies were selected and transferred to blood agar. Suspected colonies were identified as E. coli by using the VITEK II system (bioMéreiux, France). Hemolysis was also determined in blood agar. The tested isolates were stored in $20 \%$ glycerol at $-70^{\circ} \mathrm{C}$, until further characterization.

\section{Reference strains and O-serogroups}

Reference E. coli strains provided by Dr. J.M. Fairbrother (reference laboratory of E. coli, Canada) were used as positive controls for polymerase chain reaction (PCR) analysis. The strains included 7805 (F4:LT:STa:STb:EAST1:Paa), 661 (Stx1:Stx2:eae:EAST1:Paa), 1033 (F18:AIDA), 2316 (F6:STa: STb:EAST1:Paa), 13316 (F5:F41:STa:Paa), and 3463 (negative control). The O-serogroup typing was performed using the slide agglutination technique in an Animal and Plant Quarantine Agency (Korea) using rabbit antisera purchased from Serum Staten Institute (Denmark).

\section{Determination of virulence genes}

The $E$. coli genes for the toxins (LT, STa, STb, and Stx2e) and fimbriae (F4, F5, F6, F18, and F41) were amplified by PCR, which was performed using previously described protocols [4]. Bacterial colonies were suspended in $200 \mu \mathrm{L}$ of distilled water and boiled for $10 \mathrm{~min}$. After centrifugation at $8,000 \times \mathrm{g}$, the supernatant was used as a template for PCR. The reaction volume $(20 \mu \mathrm{L})$ was composed of $2 \times$ EmeraldAmp Master Mix (Takara, Japan), $2 \mu \mathrm{M}$ of each primer, and $3 \mu \mathrm{L}$ of DNA template. After amplification, the products were visualized by electrophoresis in $2 \%$ agarose gels stained with ethidium bromide.

\section{Real-time PCR assay to discriminate between F18 subtypes}

To discriminate among the F18 subtypes, a set of oligonucleotide primers and four probes were designed by comparing FedA sequences in GenBank [5]. Real-time PCR was performed using a CFX Connect Real-time PCR Detection System (Bio-Rad Laboratories, USA) with a reaction volume of $20 \mu \mathrm{L}$ that contained a $2 \mu \mathrm{L}$ template, $2 \times$ SensiMastermix

Table 1. O-serogroups and hemolytic activity of 362 Escherichia coli recovered from weaned piglets with diarrhea in Korea during 2008-2016

\begin{tabular}{|c|c|c|c|c|c|c|c|c|}
\hline \multirow{2}{*}{ O-serogroup } & \multicolumn{2}{|c|}{$2008-2010(n=143)$} & \multicolumn{2}{|c|}{$2012-2013(n=125)$} & \multicolumn{2}{|c|}{$2015-2016(n=94)$} & \multicolumn{2}{|c|}{ Total $(n=362)$} \\
\hline & No. (\%) & Hemolysis & No. (\%) & Hemolysis & No. (\%) & Hemolysis & No. (\%) & Hemolysis \\
\hline O149 & 17 (11.9) & 16 & $16(12.8)$ & 14 & $8(8.5)$ & 3 & $41(11.3)$ & 33 \\
\hline O139 & $1(0.7)$ & 1 & $15(12.0)$ & 12 & $18(19.1)$ & 14 & $34(9.4)$ & 27 \\
\hline O157 & $13(9.1)$ & 10 & $2(1.6)$ & 2 & - & - & $15(4.1)$ & 12 \\
\hline O182 & - & - & $9(7.2)$ & 2 & $4(4.3)$ & 2 & $13(3.6)$ & 4 \\
\hline O14 & - & - & $8(6.4)$ & 5 & $4(4.3)$ & 2 & $12(3.3)$ & 7 \\
\hline Others* & $50(35.0)$ & 37 & $39(31.2)$ & 22 & $15(16.0)$ & 7 & $104(28.7)$ & 66 \\
\hline OR & - & - & $23(18.4)$ & 16 & $14(14.9)$ & 11 & $37(10.2)$ & 27 \\
\hline NT & $62(43.4)$ & 36 & $13(10.4)$ & 8 & $31(33.0)$ & 21 & $106(29.3)$ & 65 \\
\hline
\end{tabular}

OR, O-rough groups (non-specific reaction); NT, not serogroupable; - , not detected. *Other serogroups (No. of total isolates): O1 (2), O2 (3), O4, O7 (2), O8 (9), O9 (3), O10 (9), O11, O20 (2), O24, O26, O28, O35 (10), O39 (2), O45, O50 (2), O51 (2), O60, O71, O73, O76 (2), O86, O91 (2), O97, (2), O98 (5), O100 (4), O101 (2), O103, O106, O107, O109 (2), O110, O111 (6), O114, O115 (2), O117, O120, O121, O127, O136 (3), O141 (2), O146 (2), O153, O154, O168, O174 (2). 
(Génepole, France), $1 \mu \mathrm{M}$ of each primer, and $0.5 \mu \mathrm{M}$ of each probe. The thermocycling profile was $95^{\circ} \mathrm{C}$ for $3 \mathrm{~min}$, followed by 40 cycles of $95^{\circ} \mathrm{C}$ for $20 \mathrm{sec}$ and $64^{\circ} \mathrm{C}$ for $25 \mathrm{sec}$.

\section{Results}

\section{Serogroups and hemolytic activity}

Table 1 shows the annual prevalence of O-serogroups and hemolytic activity of $E$. coli isolated from weaned piglets sampled during the 9 study years in Korea. Among the 51 serogroups determined in the present study, O149 (11.3\%) and $\mathrm{O} 139(9.4 \%)$ were predominant, followed by $\mathrm{O} 157$ (4.1\%), $\mathrm{O} 182(3.6 \%)$, and $\mathrm{O} 14(3.3 \%)$. The results show that the frequency of the O-serogroups changed over time. Over the 9 study years in Korea, the most prevalent serogroup in weaned piglets was O149. However, the proportion of $\mathrm{O} 139$ has increased over time, rising from $0.7 \%(2008-2010)$ to $12.0 \%$ (2012-2013) and finally $19.1 \%$ (2015-2016) prevalence. In contrast, the proportion of $\mathrm{O} 157$ has decreased over time, and in recent years (2015-2016), O157 was not detected. Interestingly, O182 and O14 were not detected from 2008 to 2010 . However, in $2012-2013$, their proportions had notably increased $(7.2 \%$ and $6.4 \%$, respectively).

Table 1 further shows the annual relationship between the O-serogroups and hemolytic activity. There were no significant differences in the relationship between serogroups and hemolytic activity over time, except for O149. The degree of association between $\mathrm{O} 149$ and hemolysis decreased over time. From 2008 to 2010 and 2012 to 2013, 16 of 17 O149 isolates (94.1\%) and 14 of 16 O149 isolates (87.5\%) showed hemolytic activity, respectively. However, from 2015 to 2016, only 3 of 8 O149 isolates (37.5\%) showed hemolytic activity. Serogroup
O139 (27 of 34 isolates, 79.4\%) and 0157 (12 of 15 isolates, $80.0 \%$ ) were highly correlated with the presence of hemolytic activity. However, O182 showed a lower association with hemolytic activity (4 of 13 isolates, 30.8\%).

\section{Adhesin and toxin genes}

The annual prevalences of $E$. coli fimbrial antigen and toxin genes isolated from weaned piglets in Korea between 2008 and 2016 are shown in Table 2. During the 9 study years, the most prevalent fimbrial antigen was F18 (43.1\%), followed by F4 $(20.2 \%)$. F18 was the most prevalent antigen in all years evaluated, accounting for 43.4\% (2008-2010), 44.0\% (20122013), and 41.5\% (2015-2016) of total prevalence. Unlike F18, the frequency of F4 and F41 changed over time. F4 was detected in 29 isolates in 2008-2010 (20.3\%) and 29 isolates in 20122013 (23.2\%). However, in 2015-2016, only 15 isolates (16.0\%) expressing F4 were detected. In contrast, F41 was only detected in 2 isolates in 2008-2010 (1.4\%) and 1 isolate in 2012-2013 $(0.8 \%)$. However, in $2015-2016,5$ isolates $(5.3 \%)$ expressing F41 were detected.

The most prevalent toxin gene in the 9 study years in Korea was STb (163 isolates, 45.0\%), followed by LT and STa (both, 121 isolates, 33.4\%). From 2012 to 2013 , the frequencies of toxin genes changed dramatically. The frequency of STb in 2012-2013 (75 isolates, 60.0\%) was higher than that detected in 2008-2010 (51 isolates, 35.7\%) as well as in 2015-2016 (37 isolates, 39.4\%). Furthermore, the frequency of Stx2e was lower in 2012-2013 (10 isolates, 8.0\%) than in 2008-2010 (51 isolates, $35.7 \%$ ) and $2015-2016$ (33 isolates, 35.1\%).

\section{Combination of toxin and adhesion}

The annual frequencies of pathotypes and virotypes

Table 2. Prevalence of fimbriae and toxin genes among 362 Escherichia coli recovered from weaned piglets with diarrhea in Korea during 2008-2016

\begin{tabular}{|c|c|c|c|c|}
\hline \multirow{2}{*}{ Fimbriae and Toxins } & \multicolumn{4}{|c|}{ No. $(\%)$ of positive $E$. coli isolates } \\
\hline & $2008-2010(n=143)$ & $2012-2013(n=125)$ & 2015-2016 (n = 94) & Total $(n=362)$ \\
\hline \multicolumn{5}{|l|}{ Fimbriae } \\
\hline F4 & $29(20.3)$ & $29(23.2)$ & $15(16.0)$ & $73(20.2)$ \\
\hline F5 & - & $2(1.6)$ & $1(1.1)$ & $3(0.8)$ \\
\hline F6 & - & - & $1(1.1)$ & $1(0.3)$ \\
\hline F18 & $62(43.4)$ & $55(44.0)$ & $39(41.5)$ & $156(43.1)$ \\
\hline F41 & $2(1.4)$ & $1(0.8)$ & $5(5.3)$ & $8(2.2)$ \\
\hline \multicolumn{5}{|l|}{ Toxins } \\
\hline $\mathrm{LT}$ & $54(37.8)$ & $55(44.0)$ & $12(12.8)$ & $121(33.4)$ \\
\hline STa & $40(28.0)$ & $51(40.8)$ & $30(31.9)$ & $121(33.4)$ \\
\hline $\mathrm{STb}$ & $51(35.7)$ & $75(60.0)$ & $37(39.4)$ & $163(45.0)$ \\
\hline Stx2e & $51(35.7)$ & $10(8.0)$ & $33(35.1)$ & $94(26.0)$ \\
\hline
\end{tabular}

-, not detected; LT, heat-labile toxin; ST, heat-stable toxin. 
(combination of toxin and fimbrial adhesion genes) are shown in Table 3. In all study years, ETEC was the predominant pathotype. There were some changes in the frequencies of pathotypes and virotypes depending on the year. The frequency of ETEC was higher from 2012 to 2013 (115 isolates, 92.0\%) than in other years. In 2008-2010, ETEC/STEC (32 isolates, $22.4 \%$ ) was detected more frequently than was STEC (23 isolates, 16.1\%). However, in 2015-2016, STEC (22 isolates, $23.4 \%$ ) was detected more frequently than was ETEC/STEC (11 isolates, $11.7 \%)$.

Virotypes in pathotypes, particularly in ETEC and ETEC/STEC, also changed over time. The most prevalent virotype in the 9 study years in Korea was F4:LT:STb (40 isolates, $11.0 \%$ ), followed by STb (39 isolates, 10.8\%), which were both in ETEC. The frequency of F4:LT:STb was higher in 2012-2013 (24 isolates, 19.2\%) than in the other years. In 20152016, the most prevalent virotype among ETEC changed to STb (9 isolates, 9.6\%), followed by STa:STb (8 isolates, $8.5 \%$ ), while the frequency of F4:LT:STb decreased ( 3 isolates, $3.2 \%$ ). Among ETEC/STEC, the most prevalent virotype was
F18:LT:STa:Stx2e in 2008-2010 (17 isolates, 11.9\%). After 2012, however, no virotype was distinctly prevalent in ETEC/STEC.

\section{F18 antigenic variants}

The annual prevalences of the F18 antigenic variants are listed in Table 4. The F18 subtype frequencies changed in a time-dependent manner. The most prevalent antigenic variant in the 9 study years in Korea was F18ac (94 isolates, 60.3\%), followed by F18ab (42 isolates, 26.9\%). F18ac was detected in 40 isolates between 2008 and $2010(64.5 \%)$ and in 36 isolates between 2012 and 2013 (65.5\%). However, from 2015 to 2016 , only 18 isolates $(46.2 \%)$ expressing F18ac were detected. The frequency of the F18new variant decreased over time, accounting for $11.3 \%$ (7 isolates, 2008-2010), 7.3\% (4 isolates, 2012-2013), and 5.1\% (2 isolates, 2015-2016) of isolates.

The relationship between the F18 subtype and pathotype also changed. Generally, F18ab isolates were primarily found in STEC, while F18ac isolates were found in ETEC. From 2008 to 2010, F18ab isolates were only detected in STEC. In 2012-

Table 3. Temporal comparison of pathotypes of 362 Escherichia coli recovered from weaned piglets in Korea during 2008-2016

\begin{tabular}{|c|c|c|c|c|}
\hline \multirow{2}{*}{$\begin{array}{l}\text { Pathotype } \\
\text { Virotype }\end{array}$} & \multicolumn{4}{|c|}{ No. (\%) of positive E. coli isolates } \\
\hline & $2008-2010(n=143)$ & $2012-2013(n=125)$ & $2015-2016(n=94)$ & Total $(\mathrm{n}=362)$ \\
\hline ETEC & $59(41.3)$ & $115(92.0)$ & $49(52.1)$ & $222(61.3)$ \\
\hline F4:LT:STb & $13(9.1)$ & $24(19.2)$ & $3(3.2)$ & $40(11.0)$ \\
\hline $\mathrm{STb}$ & $12(8.4)$ & $18(14.4)$ & $9(9.6)$ & $39(10.8)$ \\
\hline F18 & $4(2.8)$ & $16(12.8)$ & $6(6.4)$ & $26(7.2)$ \\
\hline STa:STb & - & $12(9.6)$ & $8(8.5)$ & $20(5.5)$ \\
\hline F18:STa & $4(2.8)$ & 7 (5.6) & $2(2.1)$ & $13(3.6)$ \\
\hline F18:LT:STa:STb & - & $10(8.0)$ & $1(1.1)$ & $11(3.0)$ \\
\hline F18:LT & $2(1.4)$ & $6(4.8)$ & $1(1.1)$ & $9(2.5)$ \\
\hline F18:LT:STa & - & $7(5.6)$ & $1(1.1)$ & $8(2.2)$ \\
\hline Others* & $24(16.8)$ & $15(12.0)$ & $18(19.1)$ & $57(15.7)$ \\
\hline ETEC/STEC & $32(22.4)$ & $5(4.0)$ & $11(11.7)$ & $48(13.3)$ \\
\hline F18:LT:STa:Stx2e & $17(11.9)$ & $2(1.6)$ & $2(2.1)$ & $21(5.8)$ \\
\hline F18:STa:Stx2e & $4(2.8)$ & $2(1.6)$ & $2(2.1)$ & $8(2.2)$ \\
\hline F18:LT:STa:STb:Stx2e & $3(2.1)$ & - & - & $3(0.8)$ \\
\hline F18:STa:STb:Stx2e & $1(0.7)$ & - & $2(2.1)$ & $3(0.8)$ \\
\hline Others $^{\dagger}$ & $7(4.9)$ & $1(0.8)$ & $5(5.3)$ & $13(3.6)$ \\
\hline STEC & $23(16.1)$ & $5(4.0)$ & $22(23.4)$ & $50(13.8)$ \\
\hline F18:Stx2e & $16(11.2)$ & $2(1.6)$ & $15(16.0)$ & $33(9.1)$ \\
\hline Stx2e & $7(4.9)$ & $3(2.4)$ & $6(6.4)$ & $16(4.4)$ \\
\hline F4:F18:Stx2e & - & - & $1(1.1)$ & $1(0.3)$ \\
\hline None & $29(20.3)$ & - & $12(12.8)$ & $41(11.3)$ \\
\hline
\end{tabular}

ETEC, enterotoxigenic E. coli; LT, heat-labile toxin; ST, heat-stable toxin; STEC, Shiga-like toxin-producing E. coli; -, not detected. ${ }^{*}$ Others (No. of total isolates): LT:STb (7), F4:STa:STb (7), F4:STb (6), F4 (5), F18:STa:STb (5), F18:LT:STb (4), F5:STa (3), LT:STa:STb (2), STa (2), F4:F18 (2), F41 (2), F4:F18:LT:STa:STb (2), F4:F41:LT:STa (2), F18:STb, F41:LT:STb, F4:F18:STa:STb, F6:STa:STb, F41:STa:STb, LT, F18:F41:LT:STb, F4:LT:STa. 'Others (No. of total isolates): LT:Stx2e, F4:STa:STb:Stx2e, F18:LT:Stx2e, F4:LT:STb:Stx2e, F4:LT:Stx2e, F4:STa:Stx2e, F18:LT:STb:Stx2e, LT:STa:STb:Stx2e, LT:STa:Stx2e, F18:STb:Stx2e, STa:STb:Stx2e, F4:F18:LT:STa:STb:Stx2e, F4:F41:STa:STb:Stx2e. 
Table 4. Distribution of 156 pathogenic Escherichia coli encoding F18 variants recovered from weaned piglets with diarrhea in Korea during 2008-2016

\begin{tabular}{|c|c|c|c|c|}
\hline \multirow{2}{*}{$\begin{array}{l}\text { F18 antigenic variants } \\
\text { Pathotype } \\
\text { Virotype }\end{array}$} & \multicolumn{4}{|c|}{ No. (\%) of positive $E$. coli isolates } \\
\hline & $2008-2010(n=62)$ & $2012-2013(n=55)$ & $2015-2016(n=39)$ & Total $(n=156)$ \\
\hline F18ab & $12(19.4)$ & $11(20.0)$ & $19(48.7)$ & $42(26.9)$ \\
\hline ETEC & - & $10(18.2)$ & $4(10.3)$ & $14(9.0)$ \\
\hline F18ab & - & $10(18.2)$ & $2(5.1)$ & $12(7.7)$ \\
\hline Others & - & - & $2(5.1)$ & $2(1.3)$ \\
\hline ETEC/STEC & - & - & $1(2.6)$ & $1(0.6)$ \\
\hline F18ab:STa:Stx2e & - & - & $1(2.6)$ & $1(0.6)$ \\
\hline STEC & $12(19.4)$ & $1(1.8)$ & $14(35.9)$ & $27(17.3)$ \\
\hline F18ab:Stx2e & $12(19.4)$ & $1(1.8)$ & $13(33.3)$ & $26(16.7)$ \\
\hline F18ab:F4:Stx2e & - & - & $1(2.6)$ & $1(0.6)$ \\
\hline F18ac & $40(64.5)$ & $36(65.5)$ & $18(46.2)$ & $94(60.3)$ \\
\hline ETEC & $16(25.8)$ & $32(58.2)$ & $12(30.8)$ & $60(38.5)$ \\
\hline F18ac:STa & $4(6.5)$ & $6(10.9)$ & $1(2.6)$ & $11(7.1)$ \\
\hline F18ac:LT:STa:STb & - & $9(16.4)$ & $1(2.6)$ & $10(6.4)$ \\
\hline F18ac:LT & $2(3.2)$ & $6(10.9)$ & $1(2.6)$ & $9(5.8)$ \\
\hline Others & $10(16.1)$ & $11(20.0)$ & $9(23.1)$ & $30(19.2)$ \\
\hline ETEC/STEC & $24(38.7)$ & $4(7.3)$ & $4(10.3)$ & $32(20.5)$ \\
\hline F18ac:LT:STa:Stx2e & $15(24.2)$ & $2(3.6)$ & $2(5.1)$ & $19(12.2)$ \\
\hline F18ac:STa:Stx2e & $2(3.2)$ & $2(3.6)$ & $1(2.6)$ & $5(3.2)$ \\
\hline Others & $7(11.3)$ & - & $1(2.6)$ & $8(5.1)$ \\
\hline STEC & - & - & $2(5.1)$ & $2(1.3)$ \\
\hline F18ac:Stx2e & - & - & $2(5.1)$ & $2(1.3)$ \\
\hline F18new & $7(11.3)$ & $4(7.3)$ & $2(5.1)$ & $13(8.3)$ \\
\hline ETEC & $1(1.6)$ & $3(5.5)$ & $1(2.6)$ & $5(3.2)$ \\
\hline F18new & $1(1.6)$ & $3(5.5)$ & $1(2.6)$ & $5(3.2)$ \\
\hline ETEC/STEC & $2(3.2)$ & - & $1(2.6)$ & $3(1.9)$ \\
\hline F18new:STa:STb:Stx2e & - & - & $1(2.6)$ & $1(0.6)$ \\
\hline F18new:STa:Stx2e & $1(1.6)$ & - & - & $1(0.6)$ \\
\hline F18new:STb:Stx2e & $1(1.6)$ & - & - & $1(0.6)$ \\
\hline STEC & $4(6.5)$ & $1(1.8)$ & - & $5(3.2)$ \\
\hline F18new:Stx2e & $4(6.5)$ & $1(1.8)$ & - & $5(3.2)$ \\
\hline F18nt & $3(4.8)$ & $3(5.5)$ & $1(2.6)$ & $7(4.5)$ \\
\hline ETEC & - & $3(5.5)$ & $1(2.6)$ & $4(2.6)$ \\
\hline F18nt & - & $1(1.8)$ & - & $1(0.6)$ \\
\hline F18nt:STa & - & $1(1.8)$ & - & $1(0.6)$ \\
\hline F18nt:STa:STb & - & $1(1.8)$ & - & $1(0.6)$ \\
\hline F18nt:LT:STa:STb & - & - & $1(2.6)$ & $1(0.6)$ \\
\hline ETEC/STEC & $3(4.8)$ & - & - & $3(1.9)$ \\
\hline F18nt:LT:STa:Stx2e & $2(3.2)$ & - & - & $2(1.3)$ \\
\hline F18nt:STa:Stx2e & $1(1.6)$ & - & - & $1(0.6)$ \\
\hline
\end{tabular}

ETEC, enterotoxigenic E. coli; STEC, Shiga-like toxin-producing E. coli; ST, heat-stable toxin; LT, heat-labile toxin;-, not detected; F18nt, F18 non-typeable.

2013, 32 of 36 F18ac isolates (88.9\%) were detected in ETEC. Unexpectedly, from 2012 to 2013, most F18ab isolates (10 of 11 isolates, 90.9\%) were detected in ETEC. From 2008 to 2010, F18new variant isolates were commonly detected in STEC (4 of 7 isolates, 57.1\%). However, from 2012 to 2013, 3 of 4 F18new variant isolates $(75.0 \%)$ were detected in ETEC. Some isolates were F18 non-typeable, and between 2008 and 2010, they were only detected in ETEC/STEC. In contrast, after 2012, they were only detected in ETEC. The most prevalent virotype in F18ac was F18ac:LT:STa:Stx2e (19 of 94 isolates, 20.2\%) while that 
in F18ab was F18ab:Stx2e (26 of 42 isolates, 61.9\%).

\section{Discussion}

The O-serogroup is considered one of the major virulence factors of E. coli. Although a variety of $\mathrm{O}$-serogroups has been associated with diarrhea, only a limited number of serogroups has been reported in PWD and ED [3,12,14,15,29]. In the present study, 51 O-serogroups were detected over 9 recent years in Korea, and only 5 serogroups (O149, O139, O157, $\mathrm{O} 182$, and $\mathrm{O} 14$ ) accounted for $31.7 \%$ of the typeable isolates. Among these, O139, O157, and O149 were frequently detected, as is the case in other countries. In particular, O149 and O139 are known to be associated with PWD and ED, respectively $[12,15]$.

The frequency of O-serogroups can vary from one area to another and in some specific regions, the frequency can fluctuate over time. The prevalence of and temporal changes in O-serogroups in the 9 study years in Korea are presented in Table 1. The predominant serogroup in weaned piglets over those 9 years in Korea was O149 indicating that there are ongoing risks for PWD in Korea. The O149 serogroup has been determined to be the dominant serogroup in cases of PWD in many countries including China and Canada $[8,25]$. Although only one isolate was detected in 2008-2010, the frequency of O139 isolates increased over time, and finally, the frequency of O139 was highest from 2015 to $2016(19.1 \%)$. This is in accordance with the results of Kusumoto et al. [20], which indicated that $\mathrm{O} 139$ was the predominant serogroup in Japan in 2010-2014. O139 is reported to be associated with ED, and our results indicate that the risk of ED has increased in weaned piglets in Korea. Generally, O157 can be detected in piglets worldwide [20,28] including Korea [22,23]. Kwon et al. [22] indicated that $\mathrm{O} 157$ and $\mathrm{O} 8$ were the predominant O-serogroups in Korea from 1995 to 1997 . We detected O157 in 9.1\% of isolates from 2008 to 2010 . However, no $\mathrm{O} 157$ isolates were found in 2015-2016. It is apparent that the predominant serogroup has shifted from O157 to O139 during the 9 study years on Korean farms. In O139, O182, and O14, the frequency of O-rough groups (OR) increased simultaneously. This may be due to the influence of antibiotic use. In Korea, feed-additive antibiotic growth promoters (AGPs) were frequently used. However, in July 2011, all AGPs were banned. Before this time, AGPs may have suppressed several O-serogroup antigen strains $[6,19,26]$. However, after this time, some Oserogroup-positive isolates have re-emerged.

Hemolysis provides selective advantages related to survival in the swine intestine and local environment. It is also associated with O-serogroups [24,30]. In the present study, $\mathrm{O} 149$ (80.5\%, 33/41), O139 (79.4\%, 27/34), and O157 (80.0\%, $12 / 15)$ were highly correlated with the presence of hemolytic activity. Among these, O149 demonstrated temporal changes in its relationship with hemolysis. From 2008 to 2010, 16 of 17 isolates $(94.1 \%)$ in $\mathrm{O} 149$ demonstrated hemolytic activity. However, from 2015 to 2016, just 3 out of 8 isolates (37.5\%) exhibited hemolytic activity. Although O149 is known to be closely associated with hemolytic activity [1], the present study indicates that $\mathrm{O} 149$ isolates may have no relationship with hemolysis. Moreover, this implies that the relationship between hemolysis and O-serogroups can change over time. Thus, time-dependent changes in the relationship between O-serogroups and hemolysis could affect the effectiveness of diagnosing pathogenic $E$. coli causing colibacillosis.

The detection of $E$. coli virulence factors is important for diagnosing and establishing preventative measures for colibacillosis [13]. F4 and F18 fimbriae have been frequently detected in weaned piglets in several countries such as China, Japan, Europe, and USA [9,20,24,30]. According to a previous study, the most predominant fimbrial antigen in weaned piglets in Korea was F18 [21]. In the present study, the most prevalent fimbrial antigen over the 9 study years in Korea was F18 (43.1\%) followed by F4 (20.2\%). The frequencies of F4 decreased in 2015-2016 (16.0\%) compared with that in 20122013 (23.2\%). In contrast, the frequency of F41 has increased over the years, and it accounted for 5.3\% of all isolates in 20152016. In Korea, inactivated vaccines for sows have been used countrywide. These vaccines contain E. coli whole cells with F4 and F18 [7], and such usage may have decreased the frequency of F4 and F18 and caused the emergence of new adhesin antigens such as F5, F6, and F41.

In 1999 and 2000, STa was reported as the most prevalent toxin gene in Korea [27]. However, during the 9 years of the present study, $\mathrm{STb}(44.9 \%)$ was identified as the most prevalent toxin gene followed by STa (33.4\%). This is an agreement with the results of Kusumoto et al. [20] and Zhang et al. [30] who also observed that STb was the most prevalent toxin isolated from diarrheic piglets in Japan and the USA, respectively. There were some temporal changes in the frequency of the toxin genes; the frequencies of LT, STa, and STb, which are associated with ETEC, decreased in 2015-2016 compared to those in 2012-2013. In contrast, the frequency of Stx2e significantly increased $(8.0 \%$ to $35.1 \%)$. Stx2e is a major virulence factor that causes ED $[1,16]$. These results indicate that the incidence rate of ED in weaned piglets in Korea has increased.

The clinical symptoms of colibacillosis vary depending on the pathotype of infective E. coli [28]. According to Lee et al. [23], the most predominant pathotype in Korea was EPEC. Over the 9 years of this study, ETEC has become the most predominant pathotype in weaned piglets $(61.3 \%)$. Regarding temporal changes in pathotypes, ETEC was the most prevalent pathotype over time; particularly, in 2012-2013, its frequency significantly increased (92.0\%). In July 2011, AGPs were banned in Korea. Before this prohibition, AGPs may have 
suppressed many ETEC. Subsequently, previously suppressed ETEC may have appeared suddenly, resulting in a large increase in ETEC.

Although ETEC/STEC encodes Stx2e, these strains typically induce diarrhea rather than ED. The frequencies of ETEC/ STEC have decreased from $22.4 \%$ (32 isolates, 2008-2010) to $11.7 \%$ (11 isolates, 2015-2016). In contrast, the frequency of STEC has increased to $23.4 \%$ (22 isolates) and was the second most predominant pathotype in 2015-2016. STEC is associated with ED [25], indicating an increased potential for ED occurrence in weaned piglets in Korea. The high frequency of O139 (Table 1) supports this hypothesis.

The relationship between the O-serogroup and STa:STb with unknown fimbrial adhesin remains unclear [12]. In this study, STa:STb appeared in Korea after the ban on AGPs and 8 of 20 strains $(40.0 \%)$ were from the $\mathrm{O} 182$ serogroup. This highlights the presence of a relationship between STa:STb and the specific O-serogroup O182.

E. coli contain many virulence factors appearing in various combinations. In this study, the most predominant virotype changed over time. From 2008 to 2013, F4:LT:STb and STb were the most prevalent virotypes in ETEC. In contrast, STb $(9$ isolates, 9.6\%) and STa:STb (8 isolates, $8.5 \%$ ) were the most prevalent in 2015-2016. Virotypes STb and STa:STb contain no fimbrial antigen. It may be proposed that the use of $E$. coli vaccines, which target fimbrial antigens, can cause the production of new fimbrial adhesin or non-fimbrial adhesin [7], but this has not been examined. Further studies are needed to clarify this phenomenon.

F18ab is usually associated with strains producing Stx2e (STEC), and F18ac is known to be associated with ETEC, which causes PWD [25]. In recent years, the F18new variant has been detected in Germany [2] and Korea [5]. F18ac was the most prevalent subtype in weaned piglets in the 9 years of the present study. However, the frequency of F18ac decreased over time, and in 2015-2016, its prevalence was only 46.2\%. In contrast to F18ac, the frequency of F18ab has increased over time. Based on these results, we perceive that the risk of ED in Korea is increasing.

Relationships between pathotypes and F18 subtypes have been reported [25]. Generally, F18ab isolates are primarily found in STEC and F18ac is found in ETEC [9]. We observed a similar correlation in the case of F18ac; however, in the case of F18ab, we obtained interesting results. According to Ha et al. [16], from 1996 to 2002, there was a strong correlation between F18ab and STEC. In the present study, although F18ab was exclusively detected in STEC from 2009 to 2013, 2 of 16 F18ab isolates (12.5\%) were detected in ETEC from 2015 to 2016, indicating that F18ab is also associated with ETEC. We do not know the cause of this phenomenon, but we suggest that previous antibiotic use could have affected the association of F18ab and pathotype.
From 2007 to 2016, we detected 7 isolates that were non-typeable (F18nt). The relationship between F18nt and pathotype was similar to that of F18ac. During the 9 study years, $57.1 \%$ (4/7) of F18nt isolates were in ETEC and 42.9\% (3/7) were in ETEC/STEC. Similarly, 63.8\% (60/94) of F18ac isolates were in ETEC, 34.0\% (32/94) were in ETEC/STEC, and $2.1 \%$ were in STEC. Thus, F18nt may be an antigenic variant of F18ac rather than of F18ab.

In this study, the virulence traits of $E$. coli isolated from diarrheic weaned piglets in Korea over a period of 9 years were analyzed. The results can be a source of valuable data for investigating the epidemiology of and control measures for enteric colibacillosis in Korean piggeries.

\section{Acknowledgments}

This work was supported by the Korea Institute of Planning and Evaluation for Technology in Food, Agriculture, Forestry and Fisheries (IPET) through Agri-Bio industry Technology Development Program, funded by Ministry of Agriculture, Food and Rural Affairs (MAFRA) (114058-03-3-CG000).

\section{Conflict of Interest}

The authors declare no conflicts of interest.

\section{References}

1. Baranzoni GM, Fratamico PM, Gangiredla J, Patel I, Bagi LK, Delannoy S, Fach P, Boccia F, Anastasio A, Pepe T. Characterization of Shiga toxin subtypes and virulence genes in porcine Shiga toxin-producing Escherichia coli. Front Microbiol 2016, 7, 574.

2. Barth S, Schwanitz A, Bauerfeind R. Polymerase chain reaction-based method for the typing of F18 fimbriae and distribution of F18 fimbrial subtypes among porcine Shiga toxin-encoding Escherichia coli in Germany. J Vet Diagn Invest 2011, 23, 454-464.

3. Blanco M, Lazo L, Blanco JE, Dahbi G, Mora A, López C, González EA, Blanco J. Serotypes, virulence genes, and PFGE patterns of enteropathogenic Escherichia coli isolated from Cuban pigs with diarrhea. Int Microbiol 2006, 9, 53-60.

4. Byun JW, Jung BY, Kim HY, Fairbrother JM, Lee MH, Lee WK. O-serogroups, virulence genes of pathogenic Escherichia coli and Pulsed-field gel electrophoresis (PFGE) patterns of O149 isolates from diarrhoeic piglets in Korea. Vet Med (Praha) 2013, 58, 468-476.

5. Byun JW, Jung BY, Kim HY, Fairbrother JM, Lee MH, Lee WK. Real-time PCR for differentiation of F18 variants among enterotoxigenic and Shiga toxin-producing Escherichia coli from piglets with diarrhoea and oedema disease. Vet $\mathrm{J}$ 2013, 198, 538-540.

6. Casewell M, Friis C, Marco E, McMullin P, Phillips I. The European ban on growth-promoting antibiotics and emerging consequences for human and animal health. J Antimicrob 
Chemother 2003, 52, 159-161.

7. Chae MJ, Cho JK, Lee YJ. Virulence genes of Escherichia coli isolates from piglets with diarrhea in Korea. J Anim Vet Adv 2012, 11, 9-12.

8. Chen X, Gao S, Jiao X, Liu XF. Prevalence of serogroups and virulence factors of Escherichia coli strains isolated from pigs with postweaning diarrhoea in eastern China. Vet Microbiol 2004, 103, 13-20.

9. Cheng D, Sun H, Xu J, Gao S. Prevalence of fimbial colonization factors F18ab and F18ac in Escherichia coli isolates from weaned piglets with edema and/or diarrhea in China. Vet Microbiol 2005, 110, 35-39.

10. Choi C, Cho W, Chung H, Jung T, Kim J, Chae C. Prevalence of the enteroaggregative Escherichia coli heat-stable enterotoxin 1 (EAST1) gene in isolates in weaned pigs with diarrhea and/or edema disease. Vet Microbiol 2001, 81, 65-71.

11. Duan Q, Yao F, Zhu G. Major virulence factors of enterotoxigenic Escherichia coli in pigs. Ann Microbiol 2012, 62, 7-14.

12. Fairbrother JM, Gyles CL. Colibacillosis. In: Zimmerman JJ, Karriker LA, Ramirez A, Schwartz KJ, Stevenson GW (eds.). Diseases of Swine. 10th ed. pp. 723-749, WileyBlackwell, Oxford, 2012.

13. Fairbrother JM, Nadeau E, Gyles CL. Escherichia coli in postweaning diarrhea in pigs: an update on bacterial types, pathogenesis, and prevention strategies. Anim Health Res Rev 2005, 6, 17-39.

14. Gyles CL. Fimbriae of Escherichia coli. In: Gyles CL (ed.). Escherichia coli in Domestic Animals and Humans. pp. 399-436, CAB International, Wallingford, 1994.

15. Gyles CL, Fairbrother JM. Escherichia coli. In: Gyles CL, Prescott JF, Songer JG, Thoen CO (eds.). Pathogenesis of Bacterial Infections in Animals. 4th ed. pp. 267-308, WileyBlackwell, Oxford, 2010.

16. Ha SK, Choi C, Chae C. Prevalence of a gene encoding adhesin involved in diffuse adherence among Escherichia coli isolates in pigs with postweaning diarrhea or edema disease. J Vet Diagn Invest 2003, 15, 378-381.

17. Harel J, Lapointe H, Fallara A, Lortie LA, Bigras-Poulin M, Larivière $\mathbf{S}$, Fairbrother JM. Detection of genes for fimbrial antigens and enterotoxins associated with Escherichia coli serogroups isolated from pigs with diarrhea. J Clin Microbiol 1991, 29, 745-752.

18. Imberechts H, De Greve H, Lintermans P. The pathogenesis of edema disease in pigs. A review. Vet Microbiol 1992, 31, 221-233.

19. Jensen HH, Hayes DJ. Impact of Denmark's ban on antimicrobials for growth promotion. Curr Opin Microbiol
2014, 19, 30-36.

20. Kusumoto M, Hikoda Y, Fujii Y, Murata M, Miyoshi H, Ogura Y, Gotoh Y, Iwata T, Hayashi T, Akiba M. Emergence of a multidrug-resistant Shiga toxin-producing enterotoxigenic Escherichia coli lineage in diseased swine in Japan. J Clin Microbiol 2016, 54, 1074-1081.

21. Kwon D, Choi C, Jung T, Chung HK, Kim JP, Bae SS, Cho WS, Kim J, Chae C. Genotypic prevalence of the fimbrial adhesins (F4, F5, F6, F41 and F18) and toxins (LT, STa, STb and Stx2e) in Escherichia coli isolated from postweaning pigs with diarrhoea or oedema disease in Korea. Vet Rec 2002, 150, 35-37.

22. Kwon D, Kim O, Chae C. Prevalence of genotypes for fimbriae and enterotoxins and of O serogroups in Escherichia coli isolated from diarrheic piglets in Korea. J Vet Diagn Invest 1999, 11, 146-151.

23. Lee JH, Cho HT, Kim YH, Kang HJ, Cha IH. [Isolation of enteropathogenic Escherichia coli, thermophilic Campylobacter and Salmonellae from scouring piglets]. Korean J Vet Res 1988, 28, 67-73. Korean.

24. Luppi A, Gibellini M, Gin T, Vangroenweghe F, Vandenbroucke V, Bauerfeind R, Bonilauri P, Labarque G, Hidalgo Á. Prevalence of virulence factors in enterotoxigenic Escherichia coli isolated from pigs with post-weaning diarrhoea in Europe. Porcine Health Manag 2016, 2, 20.

25. Noamani BN, Fairbrother JM, Gyles CL. Virulence genes of O149 enterotoxigenic Escherichia coli from outbreaks of postweaning diarrhea in pigs. Vet Microbiol 2003, 97, 87-101.

26. Pakpour S, Jabaji S, Chénier MR. Frequency of antibiotic resistance in a swine facility 2.5 years after a ban on antibiotics. Microb Ecol 2012, 63, 41-50.

27. Park JY, Shin NR, Park YH, Yoo HS. [Characteristics of Escherichia coli isolated from piglets with diarrhea; antimicrobial susceptibility, genotypes of enterotoxins and pili and plasmid profiles]. Korean J Vet Res 2000, 40, 301-310. Korean.

28. Paul N. Review virulence nature of Escherichia coli in neonatal swine. Online J Anim Feed Res 2015, 5, 169-174.

29. Sato JPH, Takeuti KL, Andrade MR, Koerich PKV, Tagliari V, Bemardi ML, Cardoso MRI, Barcellos DESN. Virulence profiles of enterotoxigenic Escherichia coli isolated from piglets with post-weaning diarrhea and classification according to fecal consistency. Pesq Vet Bras 2016, 36, 253-257.

30. Zhang W, Zhao M, Ruesch L, Omot A, Francis D. Prevalence of virulence genes in Escherichia coli strains recently isolated from young pigs with diarrhea in the US. Vet Microbiol 2007, 123, 145-152. 